\title{
Bioanalysis
}

\section{White Paper on recent issues in bioanalysis: focus on biomarker assay validation (BAV): (Part 3 - LBA, biomarkers and immunogenicity)}

The 2016 10th Workshop on Recent Issues in Bioanalysis (10th WRIB) took place in Orlando, Florida with participation of close to 700 professionals from pharmaceutical/ biopharmaceutical companies, biotechnology companies, contract research organizations, and regulatory agencies worldwide. WRIB was once again a weeklong event - A Full Immersion Week of Bioanalysis for PK, Biomarkers and Immunogenicity. As usual, it is specifically designed to facilitate sharing, reviewing, discussing and agreeing on approaches to address the most current issues of interest including both small and large molecules involving LCMS, hybrid LBA/LCMS, and LBA approaches, with the focus on PK, biomarkers and immunogenicity. This 2016 White Paper encompasses recommendations emerging from the extensive discussions held during the workshop, and is aimed to provide the bioanalytical community with key information and practical solutions on topics and issues addressed, in an effort to enable advances in scientific excellence, improved quality and better regulatory compliance. This White Paper is published in 3 parts due to length. This part (Part 3 ) discusses the recommendations for large molecule bioanalysis using LBA, biomarkers and immunogenicity. Parts 1 (small molecule bioanalysis using LCMS) and Part 2 (Hybrid LBA/LCMS and regulatory inputs from major global health authorities) have been published in the Bioanalysis journal, issues 22 and 23, respectively.

First draft submitted: 28 September 2016; Accepted for publication: 29 September 2016; Published online: 18 November 2016

\section{Introduction}

This year marked the 10th anniversary edition of the Workshop on Recent Issues in Bioanalysis (10th WRIB), which was held in Orlando, Florida from April 18-22, 2016. Nearly 700 professionals from pharmaceutical/biopharmaceutical companies, biotechnology companies, CROs, and regulatory agencies worldwide were in attendance. In addition to the three focused and sequential workshop days, there were six advanced training sessions throughout the week providing attendees multiple choices to combine core workshop days and training, thereby maximizing their learning process in any field of drug development expertise. As with prior WRIBs, this 10th edition was specifically designed to facilitate sharing, reviewing, discussing and agreeing upon scientific and regulatory approaches to address the most recent issues in both small and large molecule bioanalysis, biomarkers, and immunogenicity. The areas of interest included LCMS, hybrid LBA/LCMS as well as LBA/ cell-based approaches.

The chairs of the 2016 edition of the WRIB included Dr Eric Yang (GlaxoSmithKline), Dr Jan Welink (EMA), Dr An Song (Genentech), Dr Fabio Garofolo (Angelini Pharma), Dr Susan Richards (Sanofi), Dr Lakshmi Amaravadi (Sanofi) and Dr Renuka Pillutla (Bristol-Myers Squibb).

As usual, a number of regulatory agency representatives contributed actively to the 10th WRIB, including Dr Sam Haidar (US FDA), Dr Nilufer Tampal (US FDA), Dr John Kadavil (US FDA), Dr Kara Scheibner (US FDA), Dr João Pedras-Vasconcelos (US FDA), Dr Jan Welink (EU EMA), Dr Ronald Bauer (Austria AGES), Mr Jason

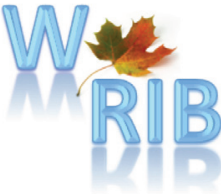

Susan Richards ${ }^{1}$, Lakshmi Amaravadi', Renuka Pillutla², Herbert Birnboeck ${ }^{3}$, Albert Torri ${ }^{4}$, Kyra J. Cowan ${ }^{5}$, Apollon Papadimitriou ${ }^{6}$, Fabio Garofolo*, , Christina Satterwhite ${ }^{8}$, Steven Piccoli², Bonnie $\mathrm{Wu}^{9}$, Corinna Krinos-Fiorotti ${ }^{10}$, John Allinson ${ }^{11}$, Flora Berisha $^{12}$, Laurent Cocea ${ }^{13}$, Stephanie Croft ${ }^{14}$, Stephanie Fraser $^{15}$, Fabrizio Galliccia ${ }^{16}$, Boris Gorovits $^{17}$, Swati Gupta ${ }^{18}$, Vinita Gupta ${ }^{5}$, Sam Haidar ${ }^{19}$, Charles Hottenstein ${ }^{20}$, Akiko Ishii-Watabe ${ }^{21}$, Darshana Jani ${ }^{17}$, John Kadavil ${ }^{19}$, John Kamerud 22, Daniel Kramer ${ }^{23}$, Virginia Litwin ${ }^{24}$, Gustavo Mendes Lima Santos ${ }^{25}$, Robert Nelson ${ }^{26}$, Yan $\mathrm{Ni}^{2}$, João Pedras-Vasconcelos ${ }^{19}$, Yongchang Qiü27, Paul Rhyne ${ }^{28}$, Afshin Safavi ${ }^{10}$, Yoshiro Saito ${ }^{21}$, Natasha Savoie ${ }^{29}$, Kara Scheibner ${ }^{19}$, Eginhard Schick ${ }^{3}$, Patricia Y. Siguenza ${ }^{5}$, John Smeraglia ${ }^{30}$, Roland F. Staack ${ }^{6}$, Meena Subramanyam ${ }^{31}$, Giane Sumner ${ }^{4}$, Theingi Thway ${ }^{32}$, David Uhlinger ${ }^{9}$, Martin Ullmann ${ }^{33}$, Alessandra Vitaliti $^{34}$, Jan Welink ${ }^{35}$, Chan C. Whiting $^{36}$, Li Xue $^{17}$ \& Rong Zeng ${ }^{37}$ *Author for correspondence: Tel.: +390691045277 f.garofolo@angelini.it

Author affiliations can be found at the end of this article.

The views expressed in this article are those of the authors and do not reflect official policy of the US FDA, Europe EMA, Health Canada, UK MHRA, The Netherlands MEB, Austria AGES, Italy AIFA, Brazil ANVISA, Japan MHLW and WHO. No official endorsement by the FDA, EMA, Health Canada, MHRA, MEB, AGES, AIFA, ANVISA, MHLW or WHO is intended or should be inferred.
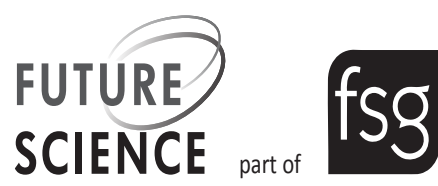


\section{Acronyms}

\begin{tabular}{|c|c|}
\hline Abbreviation & Definition \\
\hline AAPS-LBABFG & $\begin{array}{l}\text { American Association of Pharmaceutical Scientists - } \\
\text { Ligand Binding Assay Bioanalytical Focus Group }\end{array}$ \\
\hline AAPS-TPIFG & $\begin{array}{l}\text { American Association of Pharmaceutical Scientists - } \\
\text { Therapeutic Product Immunogenicity Focus Group }\end{array}$ \\
\hline ADA & Anti-drug antibody \\
\hline ADC & Antibody-drug conjugate \\
\hline ADCC & Antibody-dependent cellular cytotoxicity \\
\hline ADCP & Antibody-dependent cellular phagocytosis \\
\hline BAV & Biomarker assay validation \\
\hline BMV & Bioanalytical method validation \\
\hline CLB & Competitive ligand binding \\
\hline CLIA & Clinical Laboratory Improvement Amendments \\
\hline CLSI & Clinical and Laboratory Standards Institute \\
\hline CRO & Contract research organization \\
\hline GCC & Global CRO Council \\
\hline GLP & Good Laboratory Practices \\
\hline ICCS & International Clinical Cytometry Society \\
\hline $\mathrm{ICSH}$ & $\begin{array}{l}\text { International Council for Standardization in } \\
\text { Haematology }\end{array}$ \\
\hline ISO & International Organization for Standardization \\
\hline ISS & Incurred sample stability \\
\hline IVD & In vitro diagnostic device \\
\hline LBA & Ligand binding assay \\
\hline LCMS & Liquid chromatography mass spectrometry \\
\hline LLOQ & Lower limit of quantitation \\
\hline LPC & Low positive control \\
\hline mAb & Monoclonal antibody \\
\hline MDB & Multi-domain biotherapeutic \\
\hline MOA & Mechanism of action \\
\hline MPC & Medium positive control \\
\hline MRD & Minimum required dilution \\
\hline $\mathrm{NAb}$ & Neutralizing antibody \\
\hline PC & Positive control, used in an immunogenicity assay \\
\hline PD & Pharmacodynamic \\
\hline PK & Pharmacokinetic \\
\hline PTM & Post-translational modifications \\
\hline QC & Quality control \\
\hline RP & Reference product \\
\hline RMP & Reference medical product \\
\hline WRIB & Workshop on Recent Issues in Bioanalysis \\
\hline
\end{tabular}

Wakelin-Smith (UK MHRA), Mr Stephen Vinter (UK MHRA), Dr Fabrizio Galliccia (Italy AIFA), Mr
Gustavo Mendes Lima Santos (Brazil ANVISA), Dr Mark Bustard (Health Canada), Dr Laurent Cocea (Health Canada), Dr Akiko Ishii-Watabe (Japan MHLW-NIHS), Dr Yoshiro Saito (Japan MHLWNIHS) and Ms Stephanie Croft (WHO).

The entire workshop was designed to complete the drafting of this White Paper based on the daily working dinners and open panel discussions among the lecturers, regulators and attendees. Each core workshop day covered a wide-range of bioanalytical, biomarker and immunogenicity topics requested by members of the community, and included lectures from industry opinion leaders and regulatory representatives.

As with prior WRIB editions [1-12], a significant number of topics were addressed during the workshop and condensed into a series of relevant recommendations. In this current White Paper, the exchanges, consensus and resulting recommendations on 32 recent issues ('hot' topics) in bioanalysis, biomarkers and immunogenicity are presented. These 32 topics are distributed across the following areas:

Small molecules, peptides and small molecule biomarkers by LCMS:

- Method development challenges in bioanalysis (six topics);

- Bioanalytical regulatory challenges (five topics);

- Hybrid LBA/LCMS for biotherapeutics, biomarkers and immunogenicity:

= Biomarker and immunogenicity assays (four topics);

= PK assays (four topics);

- Large molecules by LBA and cell-based assays:

= Immunogenicity (five topics);

= Biomarkers (four topics);

- PK assays (four topics).

In addition to the recommendations on the aforementioned topics, a supplementary section has been provided in the current White Paper that specifically focuses on key inputs from regulatory agencies.

Due to its length, the 2016 edition of this comprehensive White Paper has been divided into three parts for editorial reasons. This publication (Part 3) covers the recommendations for Large Molecule Bioanalysis using LBA/cell-based assays, Biomarkers and Immunogenicity. Part 1 (Small molecules, peptides and small molecule biomarkers using LCMS) and Part 2 (Hybrid LBA/LCMS and regulatory agencies' inputs) 
are published in volume 8 of Bioanalysis, issues 22 and 23 (2016), respectively.

\section{Discussion topics}

\section{Immunogenicity}

\section{EU EMA vs US FDA clinical immunogenicity} perspective from Phase I to Phase III clinical trials Should ADA analysis be conducted in non-clinical studies and, if so, when? Is the three-tiered approach necessary for non-clinical immunogenicity testing? Regarding clinical studies, the quality and sensitivity of ADA assays can vary across the industry, resulting in immunogenicity data that cannot be directly compared. Would it be better to report clinically relevant immunogenicity data? How do we access and report the clinical relevance of an ADA response? Given the fact that any positive control is a surrogate for real ADAs, how much effort should be put into its generation and characterization? How should we translate an immunogenicity risk category (low, medium, high) into a tailored sampling/testing strategy, including specifics for each category? Could a combination of $\mathrm{ADA}$ and PK/PD serve as an alternative to dedicated neutralizing assays? Are there specific examples? Might this be acceptable from a regulatory perspective? At the recent EMA immunogenicity workshop (March 9, 2016), there was discussion on sample collection and bioanalysis that highlighted several operational questions. If samples are collected for ADA testing as per study protocol, is the sponsor obligated to analyze all the samples? If not, what scenarios would be the exceptions? In the non-clinical setting, if routine testing is not expected, is it necessary to fully validate an ADA assay for GLP testing in advance? In the event that the need for ADA testing is triggered in response to emerging data from non-clinical studies, should this work be conducted with a fully validated assay and tested under GLP, or would a qualified assay deployed in a non-GLP environment be acceptable for these investigations?

\section{Reaching industry/regulator alignment on NAb} assay format selection between cell-based assays \& non cell-based competitive ligand-binding assays What are the perspectives of regulatory agencies on $\mathrm{NAb}$ assay format selection strategy? In what cases would CLB assays be considered acceptable options for NAb detection? Is supportive data, e.g. comparison with a cell-based assay, needed to justify for this approach? Is demonstration of NAb mediated inhibition of drug binding to its target sufficient to evaluate the impact of NAb response on drug function? The $\mathrm{NAb}$ assays are expected to reflect the therapeutic

\section{Key Terms}

Immunogenicity: The ability of a substance, including biotherapeutic substances, to provoke an immune response in vivo which results in an induction of anti-drug antibodies, cytokines, etc. For the purposes of this article, ADA assays are equivalent to immunogenicity assays

Biomarker: A defined characteristic that is measured as an indicator of normal biological processes, pathogenic processes, or responses to an exposure or intervention, including therapeutic interventions. Molecular, histologic, radiographic, or physiologic characteristics are types of biomarkers. Categories of biomarkers include: susceptibility/risk biomarker, diagnostic biomarker, monitoring biomarker, prognostic biomarker, predictive biomarker, pharmacodynamic/response biomarker, and safety biomarker [FDA-NIH Biomarker Working Group. BEST (Biomarkers, EndpointS, and other Tools) Resource. BEST (Biomarkers, EndpointS, and other Tools) Resource. Silver Spring (MD): Food and Drug Administration (US); Bethesda (MD): National Institutes of Health (US); 2016].

Multi-domain biotherapeutic protein: A recombinant protein composed of domains of two (or more) different proteins and consequently able to have two (or more) functions in one protein.

Endogenous: A molecule or substance originating or produced within a biological organism or cell.

Cross-talk: Signal leakage, such as between wells of a microtiter plate

mechanism of action. Is it the regulatory expectation to examine the neutralizing capacity to all relevant functions? How can consensus be reached on what is the relevant MOA? Some therapeutic proteins contain more than one effector function, such as ADCC and ADCP. Should the assays be developed to assess NAbs to each effector function or are NAb assays specific to major functions acceptable? What aspects need to be validated vs qualified or characterized? Regarding immunogenicity test strategy, some sponsors consider not conducting NAb assessments for low risk therapeutic proteins such as monoclonal antibody biotherapeutics. Is there agreement that monoclonal antibodies, as a biotherapeutic class, always be considered low risk? What is the perspective of the regulatory agencies on not requiring NAb assessment for low risk biotherapeutics or, if required, should CLB assays be used for these low risk therapeutics as the method of choice? There continues to be an expectation that neutralizing antibody assays will be developed in support of large molecule therapeutics. Currently, ongoing discussion in industry centers around the utilization of integrated data from other assays (e.g., drug $\mathrm{PK}, \mathrm{PD}$ and $\mathrm{ADA}$ ) that may provide more sensitive methods and a comprehensive understanding of the neutralizing capacity of the detected antibodies without including the results from NAb assessments. To date, have regulators received submissions using this 
approach? How open are regulators to this approach? What is the industry experience?

Assessment of ADA classification in clinical studies: an in depth review of the current recommendations \& updated proposals based on new evidences \& case studies

What is the experience with using potential terms to describe ADA development, duration of ADA and magnitude of response? How is the relationship between time on treatment and ADA formation described? How is the duration of ADA responses defined? Are the current terms sufficient? Does the current definition of transient and persistent ADA reflect the dynamics of immune response? How do sample collection and bioanalysis contribute to these classifications? How is ADA characterized in clinical studies of short duration? How much bioanalysis is necessary? What should the sample collection duration be? What about examples where these terms are not necessarily as aligned to describe ADA duration, e.g., other ADA isotypes (IgA, $\operatorname{IgM}), 4$ versus 5 halflives (12 versus 16 weeks) for classifying persistence of IgG ADA? Are there situations where it is better to classify ADA based on median time to formation and duration of response? Which approaches are preferable for regulators and easier for treating physicians to understand the dynamics of ADA responses?

ADA/NAb assay lifecycle management $\&$ criteria for method replacement

What are the critical considerations for maintaining immunogenicity assays over time? Are the considerations for clinical development different from those during post approval? In what situations or when should we change the methods based on scientific rationale, business rationale, and risk/benefit analysis? How significant should a change be to require a new validation? What should be considered when performing bridging studies to preserve data continuity before and after method change during a study? What type of changes should trigger communication with regulatory agencies for agreement?

Evolution of biosimilar clinical immunogenicity data assessment: moving towards a consensus on the appropriate testing strategy

Which positive control should we use to assess the immunogenicity of biosimilars? How much does the selection of the positive control influence the "number" we are reporting as method sensitivity? Should one consider using multiple positive controls? What value could a standardized positive control (biosimilar) or a standardized test system offer? How are pre-existing antibodies addressed for a biosimilar when compared with the originator? Should we look for a boosted effect? Should we consider study exclusion criterion based on pre-existing antibodies (against originator)? What are the risks and benefits for this approach? Is it acceptable from a submission perspective? Is it different for Phase I and Phase III studies? Please note that discussions (positive control reagent stability and pre-existing ADA) not only are with respect to biosimilars but also cover all biotherapeutics.

\section{Biomarkers}

Global harmonization of biomarker assay

validation for regulatory submissions

Where is harmonisation lacking and what are the scientific issues? What are the current approaches and assay criteria in absence of a reference standard, presence of endogenous levels, and use of artificial matrices? What is the lesson learned on biomarker assay validation from CLIA? Is there an industry and regulator consensus on science-based criteria for clinical biomarkers for drug development submissions?

Understanding \& resolving problems \& concerns behind biomarker sample stability

QC samples are widely used and mean different things to different people. Should we clarify the different types of QCs and what they are used for (sample, analytical)? Is it necessary to monitor both spiked analytical QCs and endogenous sample QCs to establish stability? How are we managing the lack of documentation (freeze/thaw, patient detail) for samples purchased from some vendors? What samples are appropriate for use in assessing stability, individual samples or 'mega-pools'? If using 'mega-pools' to assess long-term stability, how many samples should be included in the pool to make it broadly representative of a population?

Uniqueness of flow cytometry \& measuring biomarkers in clinical development

'Fit for purpose' cellular biomarker validation: can a consensus be reached on what key parameters need to be evaluated for exploratory biomarker assays used in early clinical studies as compared with decision making biomarker assays? One of the limitations of flow based assays is the low sample stability which requires local sample analysis. How do we ensure comparability of results? With instrument alignment? Use of controls? Reference material? Technology and software improvements? Improvements in technologies and reagents are bringing a constant increase in assay 
sensitivity allowing the measurement of more and more rare cells. How can we determine and validate LLOQ in flow based assays?

\section{Multiplex biomarker assay development \& validation}

What extra requirements should be considered when comparing multiplex to singleplex assays? When is validation not necessary? What kind of rigor is needed for a biomarker assay validation to facilitate reproducibility from Phase I through Phase III? What are the key validation criteria for exploratory vs confirmatory biomarkers? In many cases, the same results are not reproduced. What is the minimum requirement to support Phase I biomarker assays for exploratory purposes so we don't doubt the results later when in Phase III. Do you agree on the importance of using matrix based controls, and not using manufacturer provided 'buffer based lyophilized controls' for precision? If yes, why? What is suggested if spiked recovery fails? What are the challenges of parallelism and dilution linearity? How will you address lot to lot changes of commercial kit contents?

\section{PK Assays}

\section{Enhancement of cross validation strategy between different LBA platforms}

What about different methods using different commercial kits? How do we address the risk of platform discontinuation? How have others addressed differences in concentrations during cross validation? What if cross validation meets criteria, yet there are still significant differences between a subset of incurred sample data? When should we worry about these differences? When does it matter and how do we address it?

Latest strategies for multi-domain biotherapeutic method development $\&$ validation in support of PK/PD

Is there a need for the detection of free/total $\mathrm{mAbs}$ and MDB? Can we control assay conditions to be certain that the analyte measured in the assay is true free/total drug? What is the industry experience with MDB biotransformation? What is the regulatory position? How can reliable MDB PK data be generated to facilitate the decision on dosing regimen? What are the main limitations of PK assays? How can these issues be overcome? Is it possible to adapt LBA platforms/formats for MDB? Are there any emerging technologies specifically designed for MDB? What are the advantages and strategies in using multiplexing assays for MDB?
Strategies to apply singlicate based sample analysis in LBA while remaining in compliance with current regulatory requirements

How can we as an industry move from a legacy driven mindset to a more science based approach? What are the road blocks? Precision will always be improved by the use of replicate samples. However, when the benefit is demonstrated to be relatively small, should singlicate analysis be considered? What is the regulatory perspective on singlicate analysis using immunoassay approaches for pharmacokinetic sample analysis? Is there any industry experience in submitting data from pharmacokinetic immunoassays that were validated using singlicate analysis?

Effective applications of incurred sample stability testing in LBA: improving confidence in data reliability

Spiked QC samples may not be able to mimic the biotherapeutic's stability in study samples due to the complexity of the biological system (ADA, isoforms, PTMs, circulating targets/receptors, etc.). Can the use of ISS for biotherapeutics enhance stability assessments for current regulatory requirements as well as provide additional information on the actual status of the samples and drug degradation? Can the use of ISS for biotherapeutics improve confidence in the reliability of PK data generated? Should there be more focus on storage and handling of samples at the clinical sites? Do you agree with the following recommendations for biotherapeutics and why: ISS studies are not typically required for most biotherapeutics as long as LTS studies during assay validation indicate sufficient analyte stability. In cases where LTS studies suggest limited biotherapeutic stability, ISS studies may be useful to extend acceptable sample stability for analysis? Do you have any real-life examples of the impact of sample processing, handling and storage on biotherapeutic ISS vs. established QC stability?

\section{Discussions, consensus \& conclusions}

There was a recurring theme that surfaced during the daily working dinners and open panel discussions, irrespective of the topic or the specific questions under discussion: "It is very important to understand the biology, the assays and their performance to be able to interpret the clinical data in context".

\section{Immunogenicity}

\section{EU EMA vs US FDA clinical immunogenicity} perspective from Phase I to Phase III clinical trials Both the EMA and FDA have issued multiple guidance documents/guidelines providing recommendations on 
the conduct of immunogenicity assays for regulatory studies, specifically the design, development and validation, to support the study sample evaluation during therapeutic protein product development.

The first guidance document from the EMA [13] was initially drafted in 2006 and came into effect in 2008. Its scope applies to proteins and polypeptides, including their derivatives, and principally addresses cases where immunogenicity is an undesired response. Two other guidelines followed in 2012 [14,15], specifically addressing $\mathrm{mAbs}$ used in clinical studies. Recently, EMA published their new updated version of the 2008 guideline [16], which is a significant revision compared with the original version in order to incorporate the substantial experience the agency has obtained from an increasing number of clinical submissions. After the consultation period was completed, a Workshop on the immunogenicity assessment of biotechnology-derived proteins was held on March 9, 2016 to discuss this draft guideline and resultant feedback.

The US FDA's guidance documents for immunogenicity testing evolved over a similar timeline. The agency's first guidance, a draft released in 2009 [17], provided recommendations on the development of immunoassays to assess immunogenicity. In 2014, FDA issued a Clinical/Medical guidance on 'Immunogenicity Assessment for Therapeutic Protein Products' [18]. Most recently, the agency updated their 2009 draft with a new draft guidance entitled Assay Development and Validation for Immunogenicity Testing of Therapeutic Protein Products [19], which was released for comments in April 2016.

These documents from the EMA and US FDA provide comparable recommendations. However, methods for examining immunogenicity have evolved over the years resulting in some notable differences in recent recommendations from the two agencies. Since clinical programs are usually global in their development, the most current recommendations from both agencies should be considered when designing an immunogenicity strategy to address multinational clinical trials and using a risk-based approach in evaluating potential safety consequences of any ADA formation.

\section{Non-clinical immunogenicity assessment}

The first issue discussed involved the scope of conducting ADA analysis in non-clinical studies. The primary aim of non-clinical immunogenicity testing is to strengthen the interpretation of toxicity results. Therefore, participants agreed that striving for the highest sensitivity is not necessary as ADA responses are readily generated when administering human biotherapeutics to animals. ADA samples should always be drawn and an ADA assay should be available. Bioanalysis should be performed in the event one observes an impact on PK exposure and detailed analysis is absolutely required if there is observed toxicity [20]. Furthermore, the reduction of collection time-points in non-clinical studies is recommended. Consensus was that the threetiered approach is not necessary. Using a screening cut point at the $99.9^{\text {th }}$ percentile is sufficient and consequently confirmatory assays and titration assays are not required. Immunogenicity assays may not always be needed if there is a good PD assay. Samples can be collected and stored if immunogenicity analysis is needed. Results may be reported as ADA positive/negative.

ADAs in toxicity studies should be assessed if there is impaired $\mathrm{PK}, \mathrm{PD}$ or toxicity observed and if one is experiencing ADA mediated adverse events occur (e.g., immune complex disease, vasculitis, anaphylaxis) [21]. For high risk molecules [22] that are identical to an endogenous counterpart, it might also be helpful to assess neutralizing antibodies in toxicity studies, as it might help to understand the consequences of crossneutralization of the endogenous molecule.

\section{Clinical immunogenicity assessment}

For clinical development programs, the quality and sensitivity of ADA assays, and how assay results are interpreted and reported, vary significantly across the industry, thereby resulting in immunogenicity data that cannot be directly compared with each other. Given this perspective, a focus should be on reporting clinically relevant immunogenicity data (impact on $\mathrm{PK} / \mathrm{PD}$ ) and adverse events. Indeed, immunogenicity as standalone information may not be too helpful if not integrated holistically. Although more immunogenicity data usually become available as the program moves through clinical development, a thorough evaluation of clinically relevant immunogenicity might only be feasible after a drug is marketed, with more patients being assessed.

There has been much discussion over the intended use of the positive control in ADA assays. Given the fact that any positive control is a surrogate for real ADAs, the amount of effort that should be put into its generation and characterization was questioned. Some characterization of this critical reagent might be required. However, it was deemed more important to have enough material for bridging strategies in case a change of positive control lot is needed. It was concluded that the positive control mainly serves as a system suitability control used to characterize the assay. A human positive control is not generally required although it can be helpful for setting up drug specific IgE assays in case of hypersensitivity reactions. Further alignment of the advantages/ limitations of either a monoclonal or polyclonal antibody control is needed. Regarding assay controls, the 
EMA recommends that a preparation of positive ADA control should ideally be used with a human preparation and available in sufficient quantities for continued use [16]. However, such controls might be very difficult to generate in practical quantities, especially for low immunogenic therapeutics.

Translation of immunogenicity risk assessment to a tailored sampling/testing strategy continues to be an area of interest. It was suggested that by creating a flow chart including multiple potential contributing immunogenicity risk factors such as route of administration, characteristics of the therapeutic molecule, formulation, etc., this approach could be helpful in guiding teams to conduct an early risk assessment which provides an initial categorization of immunogenicity risk for the molecule (low, medium, high), and translate it into an immunogenicity testing strategy. A high risk molecule results in more intensive ADA patient sampling and periodic testing (e.g., by cohort) than a low risk molecule where testing could be done at the end of a study. The scope of immunogenicity testing for medium risk molecules falls into the spectrum between frequent and non-frequent. As more information becomes available, the category of a molecule in terms of immunogenicity risk can change and the sampling/ testing strategy may need to be revised accordingly.

Regarding requirements for NAb assays, it was discussed and agreed that it is still advisable to have a NAb assay and use the totality of the PK/PD/ADA data to interpret the study data. Further investigation is needed to assess whether a suitable PD marker could be used in lieu of the NAb assay and even the binding ADA assay if it is available to predict both drug efficacy and patient safety. In general, the PK/PD/ADA information in its totality is expected to be presented together with a risk assessment to regulatory agencies.

A final discussion point regarding the latest EMA draft guidance [16] is whether the sponsor is obligated to analyze all samples collected for ADA testing. The industry seems divided on this topic since some considered that there is a scientific rationale for not analyzing all the samples. This position needs to be explained to the regulatory agencies. Moreover, at the IND/CTA stage, the regulators should be advised of the percentage of collected samples to be analysed as this may be a factor in the regulatory decision and in the communications with the sponsor. However, others concluded that all samples collected should be analyzed to get as much information as possible. Furthermore there may be an ethical consideration given samples were collected for an intended purpose. In depth industry/regulators' experience and more case studies are needed to expand the discussion on this important topic and reach a harmonized recommendation at the next WRIB.
Reaching industry/regulator alignment on NAb assay format selection between cell-based assays \& non cell-based competitive ligand-binding assays

Cell-based assays or non cell-based CLB assays may be utilized for the detection of NAbs induced by a therapeutic protein. NAbs block therapeutic function and have an immediate and direct impact on loss of drug efficacy. Neutralization of the non-redundant endogenous counterpart of the therapeutic protein may potentially result in adverse events or toxic effects due to loss of the physiological function of the endogenous protein. Detection of NAbs may therefore be an integral part of immunogenicity assessments, and reliable $\mathrm{NAb}$ assays are needed to characterize ADAs towards their neutralizing potential. The decision between using a cell-based or non cell-based assay format for $\mathrm{NAb}$ assessment should be based on a strong scientific rationale and primarily driven by the therapeutic MoA [23,24]. Cell-based assays include both bioassays and cell-based binding assays. Cell-based assays come in direct and indirect formats, with a readout based on specific downstream cellular responses that can be enhanced or reduced due to the presence of NAbs. In cell-based binding assays, NAbs are detected through the inhibition of the drug's ability to bind to the cell surface target. Non cell-based assays also come in two varieties: CLB assays which can involve either direct or indirect binding, and enzyme activity assays for enzyme biotherapeutics (e.g., enzyme replacement therapies).

Cell-based assays have traditionally been preferred by regulatory agencies. However in some situations, CLB assays have become an equally viable platform for NAb detection. For example, a CLB assay could be considered a legitimate option if the drug is an antagonistic $\mathrm{mAb}$ that blocks a soluble protein. However, if the MoA involves a cellular function that potentiates a receptor mediated signaling pathway, then a cellbased assay would be a more appropriate format. The selected assay should be justified as needed and related to the therapeutic MoA. A risk assessment should be performed during the design of NAb assays to help shape the expectation for assay performance characteristics and it is recommended to confirm the choice with the regulatory agency when there is a question. Participants agreed that a direct comparison of CLB to a cell-based assay is not mandatory for justifying the use of a CLB assay. Comparative analysis from industry sponsors has accumulated a large body of evidence in multiple case studies demonstrating that CLB assays offer comparable or even superior performance when compared with the cell-based assays [25,26]. Acceptability of the assay is based on the therapeutic MoA and 
the assay performance characteristic such as sensitivity, specificity, selectivity, precision, robustness and risk of immunogenicity.

Some therapeutic proteins exhibit multiple functionalities, such as multiple target binding, multiple effector functions, e.g., ADCC or ADCP, or have a neutralizing capacity to major biological functions with other accessory pharmaceutical effects (e.g., a $\mathrm{mAb}$ biotherapeutic blocking a cellular receptor with accessory Fc mediated ADCC function). It was discussed in depth about how many assays are needed to assess the neutralizing potential of ADA in these cases. It was agreed that the selected NAb assay should reflect the most important MoA. However, each molecule should be evaluated on a case by case basis and the strategy to be adopted should be discussed with the regulators as early as possible. It is also recommended that the qualification of the assay, instead of validation, may be acceptable during Phases I and II for low risk molecules. However, the validation needs to be completed by Phase III. For high risk molecules, a validated assay should be considered as early as Phase I.

When setting up the immunogenicity testing strategies, some scientists do not consider conducting NAb assessment for therapeutic proteins with putative low risk of immunogenicity. However, it is important to mention that the risk of immunogenicity is determined by the severity and occurrence of the clinical consequence of immune response (risk to patients) but not by the category or structural characteristics of the therapeutic protein. For example, a monoclonal antibody biotherapeutic could attain a higher risk profile if it forms aggregation or has some other quality issues that could increase the risk of immunogenicity for the drug product. The testing strategy should be determined based on the immunogenicity risk level, not on the MoA. It is advised to discuss the proposed immunogenicity testing strategy with the regulators since their perspective is that NAb should always be assessed for all biotherapeutics at the appropriate clinical development stage. In the case of low risk molecules, a validated NAb assay may not need to be implemented until Phase III.

As a follow up to the recommendation published in the 2015 White Paper in Bioanalysis [12], it was further discussed, and supported by some investigators, that the utilization of integrated data from other assays (e.g., active or target-binding competent drug PK, PD and ADA) may provide sufficient understanding of the impact of the detected ADAs on active drug exposure. $\mathrm{NAb}$ assays do not solely enable the understanding of active drug exposure in the in vivo situation, but rather characterize the ADAs with respect to their neutralizing potential. But some attendees disagreed, stating that a suitable PD marker that can quantitatively determine drug efficacy is not readily available for most of the drug development programs to date. The ADA binding assays cannot replace NAb assays to further characterize the neutralizing capacity of the ADAs which has immediate and direct impact on drug efficacy. Based on some feedback from regulatory agencies, there are still challenges to attain a clear correlation of ADA data with the clinical outcome for some biotherapeutics. Furthermore, the NAb results are not only used to address drug efficacy but also applied for the evaluation of patient safety, e.g., indicating the maturation of immune responses related to epitope spreading and assessing the clinical outcome of cross reactivity to a non-redundant endogenous counter part of the therapeutic protein. Longer sampling duration and the larger subject size during Phase III trials or even the post-marketing stage would allow more accurate correlation of NAb data to efficacy and safety. To date, regulators have received only a few submissions using this approach, and in rare cases, these submissions have been successful due to the presence of a well characterized quantitative PD marker. Unfortunately, it seems that there are not many successful cases that can accurately correlate PD data to efficacy and safety in the industry using this approach (one of the few was presented and discussed at the 9th WRIB [12]). More case studies are required in order to demonstrate to the regulators the suitability of an integrated data approach (PK, PD ADA) without including the NAb results.

In the meantime, the expectation from regulators continues to indicate that drug applications should include NAb data at the time of submission.

\section{Assessment of ADA classification in clinical} studies: an in depth review of the current recommendations \& updated proposals based on new evidences \& case studies

Evaluating the clinical effect of $\mathrm{ADA}$ requires the categorization of the antibody response observed in patients based on incidence, titer level, and other relevant factors. A commonly used characterization of ADA response is the duration of the ADA response and the association of ADA detection to clinical effects such as infusion reactions (immediate versus delayed hypersensitivity reactions) and altered PK/PD profile. To aid harmonization, a definition of terms used to describe ADA duration - transient versus persistent nature - was recently proposed [27]. This definition was based on the half-life of endogenous human IgG1, IgG2, and IgG4 and the expected clearance kinetics.

The application of the definitions proposed by Shankar et al. was illustrated with specific examples [28-30]. 
To accommodate specific immunogenicity responses that do not conform to the transient/persistent categorization, an alternate option to present data as 'median duration of ADA' was shown [27]. This option uses inter-quartile values (Q1, Q3) to describe the duration of ADA in half, $25 \%$, and $75 \%$ of the ADA positive subjects. Such an approach obviates the need to define onset or duration, but is feasible only when sample size is statistically significant. Onset is presented as the median time to antibody formation (Q2) and the inter-quartile range (Q1 and Q3). Duration is presented as the median time of antibody duration $(\mathrm{Q} 2)$ and the inter-quartile range $(\mathrm{Q} 1$ and Q3).

It was agreed to continue to harmonize reporting terms and practices for ADA classification in clinical studies with a specific focus on treatment-emergent ADA. In general, it was proposed that three terms be used: transient, persistent and last sample positive (i.e., positive ADA sample only at the last time-point of a clinical study). It was proposed that in order to properly classify the ADA duration data (transient vs persistent), at least one year of immunogenicity data is needed. It is not recommended to use these terms for shorter duration studies. In those cases, a descriptive statistical approach such as the alternate proposed above (inter-quartile values) could be used.

Additionally, in order to employ the terms adequately, it is strongly recommended to use a sufficient number of sampling time points. The sampling schedule proposed in the Shankar paper is a good starting point. For example, a sample at baseline followed by samples at 1, 2, 3, 6, 9 and 12 months followed by additional samples at 12 month intervals for longer studies may be appropriate. However, this schedule should be modified as appropriate keeping in mind that NAbs may appear later, e.g., 18 months.

The duration used to define persistent was discussed further in order to decide if 16 weeks or 12 weeks was more appropriate ( 5 versus 4 half-lives of $\mathrm{IgG}$ ). It was concluded that both can be deemed acceptable and they should be related to the ADA sampling schedule selected. Based on IgG clearance, only 6.25 and 3.13\% of the immunoglobulin remains in the body after 4 and 5 half-lives, respectively, if the immune response is not boosted.

Finally, the length of time to evaluate ADA after a subject becomes ADA positive was discussed. The recommendation is that the duration of follow up testing for ADA positive patients should be data driven and would not default to continuous monitoring until subjects returned to baseline. The latter may be hard to achieve in cases where ADA positive responses are persistent and at a high titer.
ADA/NAb assay lifecycle management \& criteria for replacement

Legacy ADA/NAb testing used to support early clinical trials are often limited by technologies and guidance available at the time of initial method development and may not meet current industry standards. As a result, these assays have presented many challenges, and the rationale for replacing legacy ADA/NAb test methods varies. Often, original ADA testing plans are very complex, using multiple technology platforms with limited tolerance to matrix interference, and have become challenging and expensive to support during routine patient care. There is also often a need to streamline the logistics of long-term patient support by outsourcing the routine ADA testing to a $\mathrm{CRO}$ with a global reach. Previous learning from immunogenicity testing to support clinical trials may show that some original testing such as isotyping is no longer useful and therefore there is no need to perform such sample analysis in post-market studies or routine patient care. Finally, with the advancement of new technologies and further understanding of immunogenicity assessments in the field, more sensitive methods are available and better defined standards and expectations for regulatory submissions. Therefore, it is best to plan ahead for any method changes. Ideally, it is generally best to try to maintain the same method throughout the drug development program unless a compelling technology driven or data driven reason justifies the need for platform switch or method modification.

It is recommended that if the assay must change during a clinical study, all samples from the study should be reanalyzed in order to ensure the data can be interpreted properly. If significant technical changes occur between trials, bridging studies should be done using retained clinical samples if informed consent covers their use. For example, a change between different LBA assay platforms, a cross comparison of the two validated methods using the same set of samples is highly recommended in order to understand whether the ADA data continuity can be maintained before and after the method change. Validation of the new assay may be preferable rather than comparing it with the old assay. Sensitivity may be different between the technologies. It is noted that differences in ADA incidence may be due to patient population and not to the change of the method if the validation is conducted appropriately. During the post-market phase, unlike during clinical trials, the cut point of the screening assay may be adjusted to minimize false positives. For routine testing to support long term patient care, the ADA testing method may be further simplified by eliminating the confirmatory assay step if it is assumed that there is no assay interference from potential 
non-specific binding to ADAs through the clinical studies. However, it was agreed that a titer assay is still necessary in order to gauge the magnitude of ADAs. A titer threshold can be identified based on statistically derived assessment of clinical relevance and ADA data higher than the threshold can be considered clinically meaningful based on the historical data. It should be noted that for some individuals, given there is generally a polyclonal response, that a titer below the threshold may still have clinical impact.

In all cases, it is recommended to have timely communication with the regulators to circumvent issues later on. The method change can be documented in the BLA, in an addendum or in a dossier along with a justification as to why the change was performed to ensure the transparency critical for BLA submission. Post-approval assay changes should also be submitted for evaluation to regulators particularly if the new assays are used to analyze post-marketing study samples.

Evolution of biosimilar clinical immunogenicity data assessment: moving towards a consensus on the appropriate testing strategy

EMA, FDA and WHO have released guidance documents addressing biosimilar immunogenicity assessments $[13,19,31]$. There is agreement that typically nonclinical immunogenicity studies are not recommended since they are considered not predictive of immunogenicity in humans. However, if conducted, the results may signal a potential problem if one should exist. Furthermore, although the FDA document does not require an Integrated Immunogenicity Summary as part of the submission (where the EMA document does), discussions during the EMA Public Workshop in London, UK in 2016 indicate that the FDA will be implementing this as well in the near future. A complete immunogenicity tiered approach is expected from biosimilar sponsors in clinical studies, including testing all confirmed ADA positive samples for NAbs, even if the innovator dossier did not originally test for NAbs.

As an update to the recommendation issued in the 2015 White Paper in Bioanalysis [12], a consensus was reached this year regarding biosimilar methods and whether a one versus two assay approach should be used. It was proposed that one assay is preferred, where the biosimilar is used as both the capture and detector reagents. This strategy presents several advantages, such as minimizing method-to-method variability, single analysis of each sample and only one method validation to execute. However, there are also disadvantages: any ADAs against immunogenic epitopes present in only the originator may not be detected.
As part of the ADA assay development, a titration of the biosimilar and originator drugs should be used to competitively inhibit the positive control in the assay. The positive control should be used at multiple levels, e.g., medium positive control (MPC) and low positive control (LPC) (refer to Figure 1), and be incubated independently with increasing amounts of biosimilar and originator drugs. The decision regarding which positive control levels should be used depends on the expected inhibition curve. The target is a curve with no or only a minor plateau so that a meaningful comparison of the antigenicity of the biosimilar and originator drugs can be performed (refer to Figure 2A \& B; the former is an example for a plateau which limits the ability to compare if this would be the only inhibition curve). In case the ADA assay will be used to measure antibodies against the biosimilar, reference product (RP) and reference medical product (RMP), all three drugs should be used in the titration experiment.

It is important to control and design the experiment carefully so that the competition curves generated by the added drugs reflect their reactivity and are not biased by the experimental conditions. Equivalent reactivity of the positive control with the biosimilar and originator can be concluded if overlapping competition curves are observed and also help justify a one-assay strategy.

Equivalent reactivity and antigenicity should be demonstrated by inhibition of a positive control with the titrated concentrations of biosimilar and reference product. Overlapping inhibition curves, by visual assessment, justify the use of the one assay strategy. If the visual assessment is inconclusive, it is recommended to use the extra sums-of-squares F-test [32]. However, if the variability of the data is large, the result of F-test will be 'not significant'. In this case, data that is not similar can be evaluated as similar. An equivalence test is another choice. US pharmacopoeia bioassay general chapters 1030, 1032, 1033, and 1034 are good references of the equivalence test [33-36].

An assessment of whether the inhibition curves of the positive control with biosimilar and originator are overlapping has the limitation at the moment they start to deviate from an obvious match. However, this does not necessarily constitute that the proof of the suitability of the positive control has failed. But in such a case, a thorough discussion of the data and justification need to be provided. Ideally, a standardized positive control for each drug substance would be used to help compare data across all development programs, not only within one company but across all those on the market as well. This is also not restricted to biosimilars but also applicable to new biotherapeutic products.

The ADA positive control is a critical reagent of all ADA assays. It was agreed that it is not necessary to 


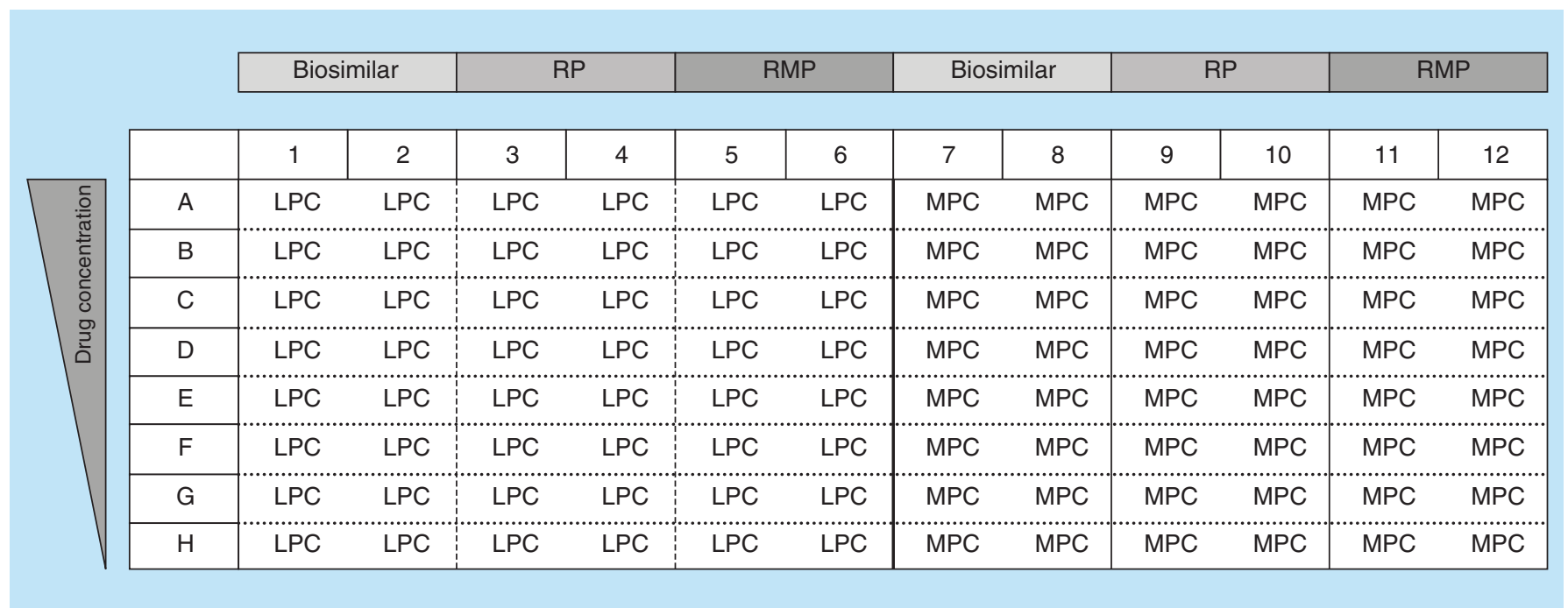

Figure 1. Example of a titration scheme to competitively inhibit the positive control.

test stability of the positive control in multiple matrices when it is used across species for both non-clinical and clinical studies. In this case, stability may only be examined in the human matrix. It is recommended to perform up to three months of stability. There is no need to go beyond that to assess long term stability as the QC samples (positive controls derived from animal species) will not yield any additional information or trends that are helpful in assessing the human ADA sample stability. This recommendation is for both biosimilars and novel biotherapeutics.

The selection of individual samples representing study samples for cut-point assessment is crucial. There was consensus that the negative control and assay cutpoint represent the biological variability of the clinical sample populations. It is recommended that, in Phase I or bioequivalence studies in naive populations, subjects with pre-existing antibodies may be screened and excluded. In Phase I, the knowledge of the prevalence of pre-existing ADA can be established early by screen- ing commercial samples and building the recruitment strategy accordingly. A high screening failure rate can be mitigated this way. As the main purpose of Phase $\mathrm{I}$ is to demonstrate bioequivalence, enrolling a 'clean' population is justifiable and not any different from the other planned stratification with exactly the same purpose. In addition, the ability to generate comparative immunogenicity data benefits from the described approach for the same reasons. However, patients with pre-existing antibodies in a Phase III study design would be representative of the patient population to be treated and therefore are not recommended to be excluded from the study. However, this does not apply if the drug label would specifically exclude treatment of patients which have been tested positive for pre-existing antibodies. In Phase III studies, which enroll a broad population who may have pre-existing antibodies, it is crucial to monitor treatment boosted levels to ensure that there are no adverse events. Subjects with pre-existing ADA should be randomized equally into the treatment arms.
(A)

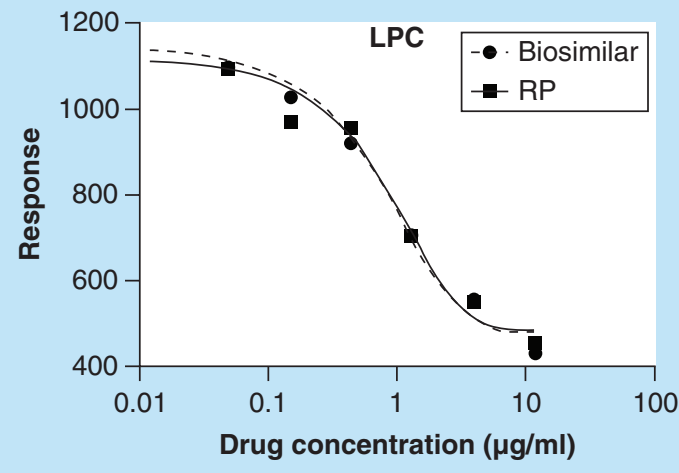

(B)

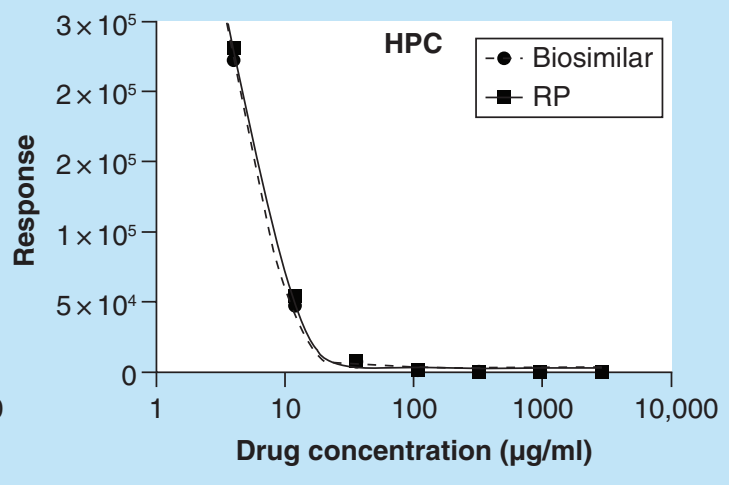

Figure 2. Examples of inhibition curves used to compare the antigenicity of the biosimilar and originator drugs. (A) Limited comparability. (B) Target curve. 
The information from sample testing using a screening ADA assay will provide this information for the randomization of subjects into the study arms. Therefore, screening the baseline samples allows a) cut-point verification against the validation cut point; this does give a head-start of $\sim 3$ weeks; and b) test for preexisting antibodies and randomization based on this information. Titering ADA assays may be of particular importance when high levels of pre-existing antibody positive subjects are present in the study. Ultimately, the desired end result in a biosimilar study is to demonstrate that there is no clinically relevant difference in immunogenicity between the biosimilar and the reference therapeutic. Study design, population and the ability to correlate the immunogenicity data with the clinical outcome are key considerations in immunogenicity assessments for biosimilar and also other biotherapeutics.

\section{Biomarkers}

\section{Global harmonization of biomarker assay} validation for regulatory submissions

Historically, clinical laboratories have the most extensive experience with diagnostic biomarkers. Indeed, CLSI has been issuing guidance on biomarker clinical assay method validation for over 40 years. Furthermore, their approach to method validation is remarkably similar to validation of methods used for bioanalysis. However, it must also be noted that the CLSI guidance was issued for a different intent. For supporting drug development needs, additional or different validation work may be needed for assays developed according to CLSI. Hence, it is recommended that scientists involved in biomarker research, as it pertains to drug development, refer to and learn from the approaches applied in the clinical laboratory community in areas where it is applicable. This recommendation is based on the fact that guidance on BMV is much younger, with the first being issued by the FDA in 2001 [37] followed by the EMA guidance a decade later [38]. These documents, however, do not include biomarkers in their scope. The first guidance to reference BAV is the recent draft FDA BMV [39]. However, discussion is still ongoing in order to improve the recommendations in that document. Several white papers have been issued in an attempt to fill this gap. The first consensus white paper came out in 2006 [40]. Additional recommendations appeared in 2012 from the European Bioanalysis Forum [41] and the Global CRO Consortium in Bioanalysis [42]. The GCC recommendations are indeed a good starting point for global BAV harmonization since they are widely used presently by the bioanalytical CRO community performing biomarker work.
Currently, there is no harmonization within industry and between regulators.

The majority of biomarkers evaluated during the drug development phase serve a variety of needs and are not directly diagnostic markers. Therefore, it was generally agreed that BAV must be fit-for-purpose and there must be an understanding of what the assay requires in terms of acceptance criteria based on the context of use and the biology. Protein biomarker calibrators made from recombinant sources do not usually reflect endogenous biomarkers. Due to this limitation, in addition to the presence of endogenous biomarker in patient samples, measurements should be considered as reflecting relative accuracy (and different from PK assays). Therefore, assay acceptance criteria should consider this fact and be flexible, matching assay validations to the requirements of the study. It is recommended, in order to ensure the most reliable validation results, using international or WHO reference materials when available. Parallelism between the protein calibrator and endogenous samples is one of the most essential parameters that should be assessed early in the lifecycle and prior to validation of a biomarker assay. It was also clear that lot-to-lot bridging experiments using QC samples were required. A final recommendation was that endogenous samples should be used for QCs and stability evaluations since they are the best approach, rather than buffer controls and surrogate QCs made from recombinant standards which provide less relevant information.

\section{Understanding \& resolving problems \& concerns} behind biomarker sample stability

The FDA guidance for monitoring biomarker sample stability has not yet been written, although CLSI and ISO guidelines have been written for this purpose with respect to reagents (not biomarkers) and are used routinely for IVD submissions. In the absence of specific FDA guidance for determining protein biomarker stability, a variety of novel solutions have arisen with the most common approach adopted from the 2001 FDA BMV [37] document, whereby samples spiked with a recombinant protein biomarker are evaluated over time to assess the biomarker 'stability'. This approach is also outlined in the 2013 draft guidance document [39]. Other approaches are briefly covered in other CLSI documents used in submissions as they serve to delimit the age of stored samples which may be used in the test.

In biomarker assays, there are two types of QCs used: endogenous QCs and spiked QCs. Spiked QCs are analytical QCs used to confirm that the calibration curve is running appropriately, prepared using recombinant material; they may or may not be in the appropriate matrix. Endogenous QCs are actual 
representative incurred samples included to represent the behavior of the study samples. At least one level of both types of QCs should be used whenever possible during sample analysis.

After an in depth discussion, consensus was attained on a different position regarding stability. It is recommended that it is not necessary to monitor stability of both the spiked analytical QCs and endogenous sample QCs. However, spiked QCs may serve as system suitability controls to aid in trouble shooting and their use is left to the discretion of the investigator. Only the endogenous sample QCs for protein biomarkers need to be shown to be stable if the analytical QCs are prepared fresh daily. If this is not possible early in drug development, it should be performed in assay validation later on in the development program. Unless it is definitively known that the recombinant reference material is identical to the endogenous protein, this approach may not adequately reflect the true stability of the endogenous sample protein. However, analytical QC stability does need to be evaluated if they are going to be stored. Freeze-thaw stability of analytical QCs may also need to be evaluated if frozen QCs may be used to support longitudinal studies.

Two case studies were brought up to support this recommendation [43]. In the first, TGF- $\beta$ QC samples underwent freeze-thaw stability experiments using spiked purified human protein and diabetic patient derived samples. Whereas stability was demonstrated in buffer using the purified protein, stability of the purified TGF- $\beta$ spiked into urine was variable and failed to meet the freeze-thaw acceptance criteria. The patient derived samples were largely intolerant to freeze-thaw cycles, with five of six samples failing to meet the stability criteria. It is worth noting that the patient samples were not analyzed as fresh samples; because of shipping, their first freeze-thaw cycle was identified as baseline.

The second case study involved the evaluation of a long-term stability of IL-13 in human serum. The recombinant protein stability was demonstrably shorter than stability of patient samples [44]. Therefore, protein biomarker stability should more optimally be established using actual individual or incurred samples so that biomarker variability induced by storage can be more precisely monitored over time. Spiked QCs do not represent incurred sample stability. Sometimes, stability will not be demonstrated until the incurred samples are available to establish stability. Until then, spiked QCs may be used to monitor the assay performance, which should be later bridged with endogenous QCs.

Managing samples purchased from vendors is an ongoing challenge due to lack of documentation on sample history (e.g., freeze-thaw, patient details). There is no evident solution available to mitigate this issue, and it remains a measured risk. It was concluded that the best that can be done is to request all the available information from the vendor or have freshly collected samples from the vendor that would mimic clinical study sample collection and avoid freeze-thaw of samples at the vendor site prior to assessing sample stability.

Finally, it was agreed that using a 'mega-pool' to assess long-term stability is not acceptable. Individual samples should be used.

\section{Uniqueness of flow cytometry \& measuring biomarkers in clinical development}

Flow cytometry is the leading technology for cellular analysis because it allows for the simultaneous detection of numerous cellular characteristics on individual cells providing a detailed understanding of cellular lineage, developmental stage, activation state, or pathology. As the pharmaceutical industry focuses more resources on cancer immunotherapy, autoimmunity, and chronic viral diseases, the importance of flow cytometry in the drug development process is increasing dramatically. With cell-depleting therapies, it is mandatory to determine efficacy and specificity of cellular depletion, and, later, repopulation. For biologics targeting cellular antigens, receptor occupancy assays are critical PD biomarkers [45-47].

The major challenges associated with using this highly complex technology in multicenter, longitudinal clinical trials include limited sample stability, cross instrument standardization, and the lack of official guidelines for the validation of flow cytometric methods [48]. Several white papers have been published in recent years to fill the regulatory gap [49-53], as well as several other guidance documents from the CLSI [54-56] and the ICSH and ICCS [57-60].

It was agreed that one of the limitations of flow based assays is the limited sample stability which in some cases may result in the requirement for sample analysis at a local bioanalytical facility or the use of special procedures at clinical sites to freeze the blood. In case of local analysis, it is important to ensure data comparability. Improvements are occurring in this area. Although there are no cellular controls specific for each method, there are some preserved controls qualified for $\mathrm{CD}^{+}{ }^{+}$and $\mathrm{CD}^{+}$cells and other major cell types which can serve as control material. Although not ideal, this material can add value depending on cell type [61]. Software is evolving and technology is rapidly advancing towards automated gating; limited tools are available to industry [62].

Advances in technology and reagents are allowing for more sensitive assays and more reliable rare event detection. Because establishing the LLOQ in flow 
cytometric assays is a challenge for very early exploratory studies, a generic LLOQ can be used (e.g., 100 cells in gate of interest). For a precise determination of LLOQ, it is recommended to dilute stained samples in unstained, or partially stained, sample and determine when the precision is no longer acceptable. Overall during sample analysis it is important to look at data and plots, the event number and the relative percentage of cells acquired. Uncertainty must be reported clearly, including the analytical variability around data points.

\section{Multiplex biomarker assay development \& validation}

Multiplex biomarker assays are used at all stages of drug development, and are increasingly being employed to measure multiple analytes in a single test, potentially saving on samples, resources, and costs. However, because of the complexity of a multiplex biomarker LBA in comparison to a singleplex assay, scientists face unique challenges while developing and validating these assays that need to be considered. This applies whether the scientist is selecting a multiplex biomarker assay kit, or designing a de novo multiplex ligand-binding assay. Some of these challenges can include cross-reactivity between reagents (depending on the reagents used and the platform); optimizing the minimum required dilution and its effects on sensitivity; lot-to-lot variability issues; and data interpretation. Validation of multiplex assays includes verifying cross-reactivity of the antibodies (specificity) and cross-talk between signal detection channels [63]. The MRD needs to be evaluated carefully; it may not be optimal for every marker in the multiplex. Sensitivity on individual analytes can also be affected by changes in buffer components. Parallelism and endogenous sample stability evaluations can pose additional challenges due to the complexity of the assays and the need for them to be assessed in a fit-for-purpose manner. For commercial multiplex assays, it is important to check the parallelism before use as non-parallelism could be an issue for the intended use in the matrix of interest. As for stability, if data are reported, it is still important to determine endogenous sample stability regardless of whether the assay is singleplex or multiplex. In order to address lot-to-lot variability of commercial kits for multiplex assays, an early assessment of the number of kits required to cover validation and sample analysis is suggested. An analysis involving the use of a correction factor or other statistical analyses can be performed [63].

It was agreed that multiplex assays for determining a very high number of analytes should very rarely be considered for regulatory use. Most multiplex assays are used experimentally to generate hypotheses. The rigor needed for validation is based on the needs of the sponsor and it should be fit-for-purpose by understanding the context and decisions required from the study. It is recommended that minimally, Phase I assay validations should have a certain level of quality; data must be reproducible. For assays used in Phase III studies, it becomes more important to incorporate disease-state samples in the validation exercises. The importance of stability and ruggedness increases. Controls around the reagents and platform used should be in place to keep assay conditions consistent. Unless data is being generated for exploratory purposes, in late stage trials it is expected that decision making biomarkers will be implemented as singleplex assays.

It is highly recommended to use matrix based endogenous QCs during the validation of multiplex assays. It is not practical to have every analyte in every sample. Adjustments may be needed depending on the value of the data and must be justified. Two schools of thought currently exist regarding the evaluation of recovery using spiked controls. Some believe that recovery of spiked samples assesses whether extraction efficiency is reproducible and consistent. Spiked recovery may help in characterization of the reference standard. An extraction efficiency of $<50 \%$ would be considered unacceptable and may require either producing reference standard material in a different system or optimizing the diluents which perturb the conformation of the reference standard. Others believe that this test has extremely limited value, providing no information about the endogenous analyte or the accuracy of the method. Parallelism is a more critical test to evaluate because accuracy is usually relative in biomarker assays. Another important consideration while validating biomarker assays is to study intra-subject variability in order to distinguish it from PD effects of the drug. It was discussed that samples from a minimum of five subjects and 3-4 time points would be good to get an estimate of intra-subject variability. Finally, each assay within a multiplexed method should be treated separately in terms of acceptance criteria. Precision of the assays will vary - often up to threefold or greater differently - and therefore setting arbitrary acceptance that is the same for all is not appropriate to ensure correct interpretation [63].

Finally, it is important that scientists involved in the development and validation of biomarker assays are engaged in the drug development teams and have a common understanding of the use of the data. There needs to be good communication across teams around the interpretation of data. Depending on the assay type (definitive, relative or quasi-quantitative), the data may need to be treated differently. 


\section{PK assays}

\section{Enhancement of cross validation strategy between different LBA platforms}

Drug development is a slow process with a high rate of failure, especially when new drug modalities are being introduced. Over the course of the lengthy drug development process, bioanalytical scientists encounter the need to bridge assay data for a variety of reasons, including 1) the need to conduct sample analysis in more than one laboratory; 2) the use of different platforms as technologies advance; and 3) simply because a commercial platform has been discontinued. These situations may warrant cross validation of methods for continuity of data within or between clinical studies. Guidance from FDA [37], EMA [38] and MHLW [64] is available as well as proposed criteria in literature [65].

The approach to cross validation when different methods using different commercial kits are bridged was discussed. Generally, this case applies to biomarker assays and rarely to PK assays. If the biotherapeutic has an endogenous counterpart and there is a commercial kit for determining the endogenous protein, it is still recommended that an in-house developed assay, with reagents generated in-house (preferred for both control and quality), which is specific for the compound, be used instead of the kit to support the long-term drugdevelopment program. If a kit has to be used, it needs to be validated for the intended purpose and the crossvalidation approaches described for bridging across different assays would apply.

Platform discontinuation is a risk affecting all methods and laboratories, as technologies continue to advance [66]. One way to reduce this risk is to diversify by using several different technologies within the lab, to avoid dependence on a single or few technology platforms. When a method needs to be moved from one platform to another due to discontinuation or changes to the platform by the vendor, cross-validation is required. Therefore, one should consider it high risk to use a single vendor technology. Ideally, a platform discontinuation should come with advance notice so that bridging experiments can be planned. It was agreed that advance notice of at least 3 to 4 years from vendors is desirable. The discontinuation of reagents is more difficult to plan for, since usually there is no advance notice or lead time. Identifying alternate sources of reagents and clearly defined criteria for bridging can be very challenging and it is difficult to provide clear recommendations for these situations.

New methods should be designed as comparable as possible to the original methods, for example, same capture and/or detection reagents when possible. Bioanalysts need to understand the tools being used and their effect on results, e.g., both methods may not be measuring the same form of the analyte. It is recommended that the same method should be used throughout the same study. If, however, the method is changed during a study and results demonstrate differences in drug concentrations when cross validating methods, correction factors are strongly discouraged. The source of the variation should be investigated and addressed before proceeding with the implementation of the new method.

If the cross validation meets criteria but there are still significant differences from a subset of incurred sample data, investigate the issue as needed. Trends or correlations should be examined, as well as how the results relate to an identifiable cause (e.g., ADA data, stability). The extent of data variation could depend on the size of the incurred sample subset as well as variability of the population. How these factors may affect the total data package and future decisions that rely on the data should be determined.

Latest strategies for multi-domain biotherapeutic method development \& validation in support of PK/PD

Many MDB, including bispecific antibodies and ADCs, have been entering drug development in recent years. The most recent indication is for cancer immunotherapy, where bispecific mAbs are engineered to simultaneously bind to a cytotoxic cell using, for example, CD3 receptor and a tumor cell target [67-69]. The specificity of independent functional domains determines the compound's MoA. Bioanalysis of these new generation biologics introduces additional requirements when assessing assay characteristics and during data interpretation. Previously raised questions regarding the analysis of the total vs free form of the drug [9] is even more critical for bispecific antibodies. When developing an assay, a complexity-based approach on the therapeutic MoA should be used. The structure of the molecule should be considered, e.g., whether it is a full-length or $\left.\mathrm{F}(\mathrm{ab})_{2}\right)_{2}$ antibody. Also, the affinity of each arm of such a bispecific should be considered, as well as the biology and what the PK analysis needs are. Measuring the active form of the drug is ideal but it is not always possible to have a 'true' active assay [70]. A total assay can be used when additional information to help evaluate the results is available [71,72]. The comparative outcome of the total analyte assay versus that for an active analyte depends on the relative to the drug concentration of the binding target and drug-target binding affinity. These two parameters may help to appropriately model how the total analyte concentration may reflect on the measurement of the desired drug analyte. Availability of the total or free 
target concentration values as well as understanding of the binding affinity is therefore important to evaluate drug PK assay results.

Analysis of the impact of compound biotransformation, including instability in circulation on the final PK assay outcome and decisions on dosing regimen, is important for ADC modalities. Industry experience with MDB biotransformation is still fairly limited and no data has been reportedly reviewed by regulators to date. There is no guarantee that reliable MDB data for PK analysis can be generated due to the technical challenges. Bioanalysts can only characterize the assay to the best of their abilities with the tools at their disposal, as with all bioanalytical methods. Multiple assays for MDBs may be needed depending on reagent availability. It is recommended for the assays to demonstrate that target binding for MDBs is intact for the individual domains.

LBA platforms and formats can be adapted to MDB analyses. For example, it is possible to use emerging multiplex platforms even though they are not specifically designed for MDB. One of the advantages of a multiplex assay is the ability to perform the necessary measurements as part of a single assay with a limited sample volume. However, for MDBs, it must be demonstrated that the technology is not biased towards measurement of one component over the other. Also, it may be more difficult and require a bigger effort in assay development, reagent selection, long term supply and validation (e.g., interferences, specificity, dynamic range, etc.). Understanding what information is most critical for the PK and toxicity assessment is highly important when designing an appropriate PK assay format and approach.

\section{Strategies to apply singlicate based sample} analysis in LBA while remaining in compliance with current regulatory requirements

The practice of performing bioanalytical sample analysis using immunoassay techniques as replicates, typically in duplicate, originates from an attempt to normalize the inherent variability of the assay from relatively minor errors, as well as to identify and exclude data from the more infrequent gross errors that might arise from issues associated with reagent quality, analyst capability, or other identifiable causes in the analytical process. However, based on experience, the most meaningful repeat analysis is not due to differences in replicates, but attributed to other factors either biological or analytical. Current regulatory views on the use of replicate analysis are mixed. The 2001 FDA BMV [37] only recommends it for 'difficult procedures'. Singlicate assays are permitted if the pre-study assay validation demonstrates acceptable variability. The revised draft guidance [33] does not comment on the necessity of replicate analysis of study samples. In contrast, the EMA and Japanese guidance documents $[38,64]$ recommend using replicates to assay study samples. Literature outlining past industry experience has recommended using replicate analysis as well [73,74]. However, improvements in reagent quality and increasing use of automation in both sample preparation and analytical platforms have significantly improved assay reproducibility and made the use of single replicate sample analysis by immunoassay more acceptable.

The recommendation for using singlicate analysis for immunoassay techniques does not apply to every method. However, once there is confidence in an assay, e.g., low failure rates with adequate precision, there should be no problem to move to a singlicate sample analysis approach. It was agreed that there are no road blocks to the use of singlicates for immunoassay techniques used for PK analysis, only if the bioanalytical immunoassay method demonstrates acceptable reproducibility and robustness to meet predefined performance criteria. In fact, there has been industry experience, confirmed by regulators, that acceptable submissions have been performed using immunoassay techniques in singlicate that were demonstrated to be robust and reproducible over the course of drug development by analytical validation and preclinical and clinical study support which included reproducible ISR, sample parallelism and PK sample repeat results.

Effective applications of incurred sample stability testing in LBA: improving confidence in data reliability

Analyte stability is a critical parameter in the validation of a bioanalytical procedure. For LBA, ISS studies can be used for investigational purposes and are not currently required by regulatory agencies $[6,10,75]$. In most cases, stability studies conducted with spiked samples during method validation are sufficient to demonstrate the stability of the analyte in the matrix of interest for PK assays.

From a regulatory point of view, accountability and documentation for sample handling and storage should be a focal point because of the importance of sample integrity. Analysts should understand how samples are being handled and should ensure that any stability related issues can be addressed in method validation. It was acknowledged that there are cases where the analyte either has potential stability issues or is only available as a recombinant protein that may not exhibit the same stability as the native protein. In addition, analyte in circulation may undergo modifications or can be bound to various matrix components 
which may make the protein less stable in the matrix due to the complexity of the biological system (ADA, isoforms, PTMs, circulating targets/receptors, proteolysis, glycation, etc.). On the other hand, depending on the form of the biotherapeutic that is being measured (for example in a free PK assay), there may be changes in measured concentration upon storage due to differences in association/dissociation with other interacting components such as the target or ADA. Hence, due to in vivo modifications of the analyte ISS may differ from the stability of a spiked QC sample. In some cases, conducting the ISS evaluation may be helpful to establish incurred sample stability.

\section{Recommendations}

Below is a summary of the recommendations made during the 10th WRIB.

\section{Immunogenicity}

1. For ADA analysis in non-clinical studies, striving for the highest sensitivity is not necessary. A screening cut point at the 99.9th percentile is preferred and consequently confirmatory assays and titration assays are not required. Detailed ADA analysis should be conducted if impaired PK/PD, toxicity or ADA related adverse events are observed. For high risk molecules with an endogenous counterpart, it might also be helpful to assess neutralizing antibodies to evaluate the consequences of crossneutralization of this endogenous molecule.

2. For NAb assays for multi-domain proteins that contain more than one effector function, e.g., ADCC and $\mathrm{ADCP}$, or $\mathrm{mAb}$ biotherapeutics having a neutralizing capacity to a major biological function, one assay that reflects the primary therapeutic MoA is needed for NAb assessment. For example, NAb assay specific to the ADCC function would be sufficient to biotherapeutics with both ADCC and ADCP function. However, the NAb assay strategy should be assessed and determined on a case by case basis. The regulatory agencies should be consulted for decision making when there are questions. During early drug development (Phase I and Phase II), NAb assays are generally not required for low risk molecules. If a NAb assay is desired for early phase drug development of a low risk biotherapeutic, then qualification of the assay, instead of validation, would be sufficient. However, a validated NAb assay is required for Phase III studies of a low risk molecule. For high risk biotherapeutics, a validated assay could be considered in Phase I for $\mathrm{NAb}$ assessment to evaluate drug safety. Utiliza- tion of integrated data from other assays (e.g., drug $\mathrm{PK}, \mathrm{PD}$ and $\mathrm{ADA}$ ) that may provide more sensitive methods and a comprehensive understanding of the clinical impact of detected ADA without including the results from NAb assay can be scientifically discussed with regulatory agencies and are encouraged by industry sponsors. However, the expectation from regulators continues to be that NAb data should be included in the package at the time of submission. There may be valid reasons for accepting submissions without NAb data (e.g., appropriate biomarker data available, some oncology indications).

3. To illustrate and describe ADA duration, a graphical option can be considered when sample size is statistically significant. This option presents data as "median duration of ADA" and uses inter-quartile values $(\mathrm{Q} 1, \mathrm{Q} 3)$ to describe the duration of $\mathrm{ADA}$ in half, $25 \%$, and $75 \%$ of the ADA positive subjects. Moreover, to properly classify the ADA duration data, at least one year of immunogenicity data is recommended. To employ the terms adequately, a sufficient number of time points should also be used to opportunely modify the sampling schedule proposed in Shankar's paper while keeping in mind that NAbs may appear later. A 16 week or 12 week duration are both deemed acceptable to characterize the patient as having an ADA as persistent response and an adequate sampling schedule should be selected. The duration of follow up testing for ADA positive patients should be data driven and should not default to continuous monitoring until subjects become baseline negative.

4. For NAb/ADA assay lifecycle management, if the assay must be changed within a clinical study, all samples should be reanalyzed using the new method in order to ensure the data can be interpreted properly as a whole. If changes are required between trials, bridging studies may be appropriate using retained clinical samples. If there is a significant technology change, for example a change between different LBA assay platforms or $\mathrm{NAb}$ assays based on a different therapeutic MoA, a cross comparison of the two validated methods using the same set of patient samples are highly recommended in order to understand whether the ADA data continuity can be maintained before and after the method change. Assay changes occurring during the post-market phase do not typically require a tiered approach and screening and titer assays would be sufficient, without the need to conduct the confirmation assay. 
5. For biosimilar clinical immunogenicity data assessment, one assay is preferred in which the biosimilar is used as both the capture and detector reagent.

6. When the ADA positive control is used across species for both non-clinical and clinical studies, it is not necessary to test stability in multiple matrices; only the stability data in human matrix is generally needed. In addition, three months of stability in the human matrix instead of long term stability is typically sufficient. A long term stability assessment with the positive control antibody does not add value and therefore is not required for both biosimilar and new biotherapeutics. For bioequivalence studies, the positive control can be developed against the innovator or biosimilar. For practical reasons, development of the positive control might be started early with the innovator as representative and the fully characterized material of the biosimilar will become available later in the development process. In the selection of individual samples for cut-point assessment, the selected matrix samples must represent the biological variability of the clinical sample population. In Phase III studies, treatment boosted ADA levels should be monitored to ensure that there are no correlated adverse events. Clearly, this is not restricted to biosimilars but also applicable to novel biotherapeutic products.

\section{Biomarkers}

1. The bioanalytical community can refer to the practices and learn from clinical laboratory guidelines in their approaches to BAV in applicable areas. The GCC recommendations are a good starting point for BAV global harmonization. BAV must be fitfor-purpose and endogenous samples should be used as QCs rather than buffer controls and surrogate QCs as much as possible to evaluate assay validation parameters.

2. For biomarkers, it is not necessary to monitor stability of both the spiked analytical QCs and endogenous sample QCs. Only the endogenous sample QCs need to be shown to be stable for protein biomarkers. Moreover, biomarker stability should be supported by using incurred samples so that biomarker variability induced by storage can be more precisely monitored over time. Individual samples should be used for assessing stability of biomarker in the assay and the use of a 'mega-pool' to assess long-term stability is not applicable.

3. The low sample stability is one of the major limitations of flow cytometry assays. To improve com- parability between laboratories some preserved controls qualified for CD4s, CD8s and other major cell types can be used, even if not yet ideal. When examining samples, it is necessary to look at data and plots, the event number and the relative percentage of cells acquired. It is necessary to identify where precision is no longer acceptable. Uncertainty must be reported clearly, including the analytical variability around data points.

4. Multiplex assays with a very high number of analytes should very rarely be considered for validation. However, the performance characteristics of the assay should be understood by employing a fitfor-purpose validation approach based on understanding of the context of how the assay will be used and the decisions to be made. Cross-reactivity of the antibodies and cross-talk between signal detection channels should always be verified during assay development. The MRD needs to be evaluated carefully since it may not be optimal for every marker in the multiplex and greatly affect sensitivity. Matrix based endogenous QCs are highly recommended to be used during the validation of biomarker assays. Parallelism testing should be performed because accuracy is usually relative in biomarker assays. Scientists involved in biomarker assay development and validation should be engaged in the drug development teams and they need to have a common understanding of the use of the data and how the data should be interpreted (i.e., absolute values, fold change, change from baseline, etc.).

\section{PK assays}

1. In order to mitigate the risk of platform discontinuation or catastrophic loss, laboratories should employ several different technologies and limit the use of single vendor technologies. Platform discontinuation should have at least a 3 to 4 year advanced notice from vendors. When results demonstrate differences in concentrations when cross validating methods, the use of correction factors is discouraged. If the cross validation meets criteria but there are still significant differences between a subset of incurred sample data, the issue should be investigated as needed. Trends or correlations should be examined, as well as how the results relate to an identifiable cause.

2. When developing an assay for MDB, a complexitybased approach according to the therapeutic MoA should be used. Multiple assays may be needed 
depending on the reagents available. It should be demonstrated that target binding is intact for the individual domains. LBA platforms and formats can be adapted to MDB. Emerging multiplex platforms are encouraged even though they are not specifically designed for measuring MDB as part of a single assay with a limited sample volume if deemed appropriate.

3. The use of singlicate sample analysis for immunoassay techniques does not necessarily apply to every type of LBA. However, once the precision of the method has been demonstrated through validation and there is confidence in the assay, then it is reasonable to move forward with a singlicate approach for PK sample analysis. Overall, there should be no issues regarding the singlicate approach in methods that were demonstrated to be robust during prestudy validation.

4. The use of ISS rather than traditional spiked QC samples may be considered in cases where a biotherapeutic found in an incurred study sample may have a potential stability issue or is only available as a recombinant protein that may not exhibit the same stability as the native protein. In addition, ISS should be considered when the analyte in circulation may undergo modifications or binding events which may alter the protein and make it less stable

'Sanofi, Framingham, MA, USA

${ }^{2}$ Bristol-Myers Squibb, Princeton, NJ, USA

${ }^{3}$ Roche Pharma Research and Early Development, Roche Innovation

Center, Basel, Switzerland

${ }^{4}$ Regeneron Pharmaceuticals, Tarrytown, NY, USA

${ }^{5}$ Genentech, South San Francisco, CA, USA

${ }^{6}$ Roche Pharma Research and Early Development, Roche Innovation

Center, Munich, Germany

${ }^{7}$ Angelini Pharma, Pomezia, RM, Italy

${ }^{8}$ Charles River, Reno, NV, USA

9 Janssen R\&D, Spring House, PA, USA

${ }^{10}$ Bioagilytix Labs, Durham, NC, USA

${ }^{11}$ LGC, Cambridge, UK

${ }^{12}$ Daiichi Sankyo, Edison, NJ, USA

${ }^{13}$ Health Canada, Ottawa, ON, Canada

${ }^{14} \mathrm{WHO}$, Geneva, Switzerland

${ }^{15}$ Pfizer, Groton, CT, USA

${ }^{16}$ Italy AIFA, Rome, Italy

${ }^{17}$ Pfizer, Andover, MA, USA

${ }^{18}$ Allergan, Irvine, CA, USA

\section{References}

1 Savoie N, Booth BP, Bradley T et al. 2008 White Paper: the 2nd calibration and validation group workshop on recent issues in good laboratory practice bioanalysis. Bioanalysis 1(1), 19-30 (2009).

2 Savoie N, Garofolo F, van Amsterdam P et al. 2009 White Paper on recent issues in regulated bioanalysis from the $3 \mathrm{rd}$ in the matrix due to the complexity of the biological system.

\section{Acknowledgements}

The authors would like to acknowledge the US FDA, Europe EMA, UK MHRA, Austria AGES, Italy AIFA, Brazil ANVISA, Health Canada, Japan MHLW and WHO for supporting this workshop. S Richards (Sanofi), L Amaravadi (Sanofi/ Genzyme), R Pillutla (Bristol-Myers Squibb), H Birnboeck (F. Hoffmann-La Roche Ltd.), F Garofolo (Angelini Pharma) for chairing the workshop and/or the white paper discussions. All the workshop attendees and members of the bioanalytical community who have sent comments and suggestions to complete this White Paper. W Garofolo, L Lu, X Wang, M Losauro, N Savoie, A Hernandez, K Kalaydjian and J Conception for the assistance in the organization of the event. Future Science Group as a trusted partner.

\section{Financial \& competing interests disclosure}

The authors have no relevant affiliations or financial involvement with any organization or entity with a financial interest in or financial conflict with the subject matter or materials discussed in the manuscript. This includes employment, consultancies, honoraria, stock ownership or options, expert testimony, grants or patents received or pending, or royalties.

No writing assistance was utilized in the production of this manuscript.

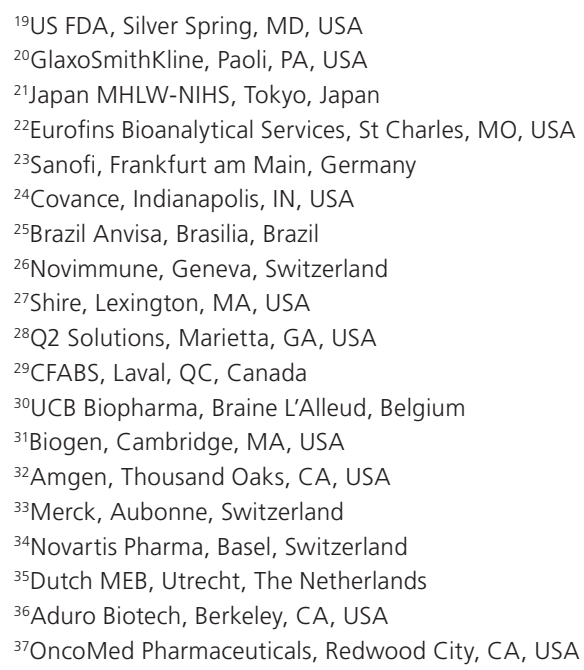

calibration and validation group workshop. Bioanalysis 2(1), 53-68 (2010).

3 Savoie N, Garofolo F, van Amsterdam P et al. 2010 White Paper on recent issues in regulated bioanalysis and global harmonization of bioanalytical guidance. Bioanalysis 2(12), 1945-1960 (2010).

4 Garofolo F, Rocci M, Dumont I et al. 2011 White Paper 
on recent issues in bioanalysis and regulatory findings from audits and inspections. Bioanalysis 3(18), 2081-2096 (2011).

5 DeSilva B, Garofolo F, Rocci M et al. 2012 White Paper on recent issues in bioanalysis and alignment of multiple guidelines. Bioanalysis 4(18), 2213-2226 (2012).

6 Stevenson L, Rocci M, Garofolo F et al. 2013 White Paper on recent issues in bioanalysis: 'Hybrid' - the best of LBA \& LC/MS. Bioanalysis 5(23), 2903-2918 (2013).

7 Fluhler E, Hayes R, Garofolo F et al. 2014 White Paper on recent issues in bioanalysis: a full immersion in bioanalysis (part 1 -small molecules by LCMS). Bioanalysis 6(22), 3039-3049 (2014).

8 Dufield D, Neubert, H, Garofolo F et al. 2014 White Paper on recent issues in bioanalysis: a full immersion in bioanalysis (part 2 - hybrid LBA/LCMS, ELN \& regulatory agencies' input). Bioanalysis 6(23), 3237-3249 (2014).

9 Stevenson L, Amaravadi L, Myler H et al. 2014 White Paper on recent issues in bioanalysis: a full immersion in bioanalysis (part 3 - LBA and immunogenicity). Bioanalysis 6(24), 3355-3368 (2014).

10 Welink J, Fluhler E, Hughes N et al. 2015 White Paper on recent issues in bioanalysis: focus on new technologies and biomarkers (part 1 - small molecules by LCMS). Bioanalysis 7(22), 2913-2925 (2015).

11 Ackermann B, Neubert H, Hughes N et al. 2015 White Paper on recent issues in bioanalysis: focus on new technologies and biomarkers (part 2 - hybrid LBA/LCMS and input from regulatory agencies). Bioanalysis 7(23), 3019-3034 (2015).

12 Amaravadi L, Song A, Myler H et al. 2015 White Paper on recent issues in bioanalysis: focus on new technologies and biomarkers (part 3 - LBA, biomarkers and immunogenicity). Bioanalysis 7(24), 3107-3124 (2015).

13 European Medicines Agency. Committee for Medicinal Products for Human Use (CHMP). Guideline on immunogenicity assessment of biotechnology-derived therapeutic proteins. London, UK (2007). EMEA/ CHMP/BMWP/14327/2006. www.ema.europa.eu/docs/ en_GB/document_library/Scientific_guideline/2009/09/ WC500003946.pdf

14 European Medicines Agency. Committee for Medicinal Products for Human Use (CHMP). Guideline on immunogenicity assessment of monoclonal antibodies intended for in vivo clinical use. London, UK (2012). EMA/ CHMP/BMWP/86289/2010. www.ema.europa.eu/docs/ en_GB/document_library/Scientific_guideline/2012/06/ WC500128688.pdf

15 European Medicines Agency. Committee for Medicinal Products for Human Use (CHMP). Guideline on similar biological medicinal products containing monoclonal antibodies - non-clinical and clinical issues. London, UK (2012). EMA/CHMP/BMWP/403543/2010. www.ema. europa.eu/docs/en_GB/document_library/Scientific_ guideline/2012/06/WC500128686.pdf

16 European Medicines Agency. Committee for Medicinal Products for Human Use (CHMP). Draft guideline on Immunogenicity assessment of biotechnology-derived therapeutic proteins. London, UK (2015). EMEA/CHMP/
BMWP/14327/2006 Rev.1. www.ema.europa.eu/docs/ en_GB/document_library/Scientific_guideline/2015/10/ WC500194507.pdf

17 US Department of Health and Human Services, US FDA, Center for Drug Evaluation and Research, Biologics Evaluation and Research. Draft Guidance for Industry, Assay Development for Immunogenicity Testing of Therapeutic Proteins. Rockville, MD, USA (2009). www.bioagilytix. com/wp-content/uploads/2016/02/FDA-GUIDANCEAssay-Development-Immunogenicity-Testing.pdf

18 US Department of Health and Human Services, US FDA, Center for Drug Evaluation and Research, Biologics Evaluation and Research. Guidance for Industry, Immunogenicity Assessment for Therapeutic Protein Products. Rockville, MD, USA (2014). www.fda.gov/downloads/ drugs/guidancecomplianceregulatoryinformation/guidances/ ucm338856.pdf

19 US Department of Health and Human Services, US FDA, Center for Drug Evaluation and Research, Biologics Evaluation and Research, Center for Devices and Radiological Health (CDRH). Draft Guidance for Industry, Assay Development and Validation for Immunogenicity Testing of Therapeutic Protein Products. Rockville, MD, USA (2016). www.fda.gov/downloads/Drugs/./Guidances/UCM192750. pdf

20 Ponce R, Abad L, Amaravadi L et al. Immunogenicity of biologically-derived therapeutics: assessment and interpretation of nonclinical safety studies. Regul. Toxicol. Pharmacol. 54(2),164-182 (2009).

21 ICH Harmonised Tripartite Guideline S6(R1). Preclinical safety evaluation of biotechnology-derived pharmaceuticals (2011). www.ich.org/fileadmin/Public_Web_Site/ ICH_Products/Guidelines/Safety/S6_R1/Step4/S6_R1_ Guideline.pdf

22 European Medicines Agency. Committee for Medicinal Products for Human Use (CHMP). Draft Guideline on Requirements for First-In-Man Clinical Trials for Potential High-Risk Medicinal Products. London, UK (2007). EMEA/ CHMP/SWP/28367/2007 Corr. www.ema.europa.eu/docs/ en_GB/document_library/Scientific_guideline/2009/09/ WC500002989.pdf

23 USP-NF. General Information, <1106.1> Immunogenicity Assays - Design and Validation of Assays to Detect Anti-drug Neutralizing Antibodies.

24 Wu B, Chung S, Jiang XR et al. Strategies to determine assay format for the assessment of neutralizing antibody responses to biotherapeutics. AAPS J. doi: 10.1208/s12248-016-9954-6 (2016) (Epub ahead of print).

25 Wu BW, Gunn GR, Shankar G. Competitive ligandbinding assays for the detection of neutralizing antibodies. In: Detection and Quantification of Antibodies to Biopharmaceuticals: Practical and Applied Considerations. Tovey MG (Ed.). John Wiley \& Sons, Inc. Hoboken, NJ, USA (2011).

26 Finco D, Baltrukonis D, Clements-Egan A et al. Comparison of competitive ligand-binding assay and bioassay formats for the measurement of neutralizing antibodies to protein therapeutics. J. Pharm. Biomed. Anal. 54(2), 351-358 (2011). 
27 Shankar G, Arkin S, Cocea L et al. Assessment and reporting of the clinical immunogenicity of therapeutic proteins and peptides-harmonized terminology and tactical recommendations. AAPS J. 16(4), 658-73 (2014).

28 Subramanyam M. 'Case Study: Immunogenicity of natalizumab.' In: Immunogenicity of Biopharmaceutics. van de Weert M, Horn Møllier E (Eds). AAPS Books Web, Springer, NY, USA, 173-187 (2008).

29 Steenholdt C. Transient and persistent antibodies against TNF-inhibitors in IBD. Am. J. Gastroenterol. 110, 16231624 (2015).

Zhou L, Hoofring SA, Wu Y et al. Stratification of antibody positive subjects by antibody level reveals an impact of immunogenicity on pharmacokinetics. AAPS J. 15(1), 30-40 (2013).

31 World Health Organization. Guidelines on Evaluation of Similar Biotherapeutic Products (SBPs). WHO Expert Committee on Biological Standardization, Sixtieth report (1923 October 2009). WHO Technical Report Series No. 977, 2013 - Annex 2 (2013). http://apps.who.int/medicinedocs/ documents/s19941en/s19941en.pdf

32 Marini JC, Anderson M, Cai XY et al. Systematic verification of bioanalytical similarity between a biosimilar and a reference biotherapeutic: committee recommendations for the development and validation of a single ligand-binding assay to support pharmacokinetic assessments. AAPS J. 16(6), 1149-1158 (2014).

USP-NF. General Information, <1030> biological assay chapters - overview and glossary (2013).

34 USP-NF. General Information, <1032> design and development of biological assays (2012).

35 USP-NF. General Information, <1033> validation of biological assays (2012).

36 USP-NF. General Information, <1034> analysis of biological assays (2012).

37 US Department of Health and Human Services, US FDA, Center for Drug Evaluation and Research, Center for Veterinary Medicine. Guidance for Industry, Bioanalytical Method Validation, Rockville, MD, USA (2001). www.fda. gov/downloads/Drugs/Guidance/ucm070107.pdf

38 European Medicines Agency. Committee for Medicinal Products for Human Use (CHMP). Guideline on Bioanalytical Method Validation. EMEA/CHMP/ EWP/192217/2009. London, UK (2011). www.ema. europa.eu/docs/en_GB/document_library/Scientific_ guideline/2011/08/WC500109686.pdf

39 US Department of Health and Human Services, US FDA, Center for Drug Evaluation and Research, Center for Veterinary Medicine. Draft Guidance for Industry, Bioanalytical Method Validation. Rockville, MD, USA (2013). www.fda.gov/downloads/drugs/ guidancecomplianceregulatoryinformation/guidances/ ucm368107.pdf

40 Lee JW, Devanarayan V, Barrett YC et al. Fit-for-purpose method development and validation for successful biomarker measurement. Pharm. Res. 23(2), 312-328 (2006).

41 Timmerman P, Herling C, Stoellner D et al. European
Bioanalysis Forum recommendation on method establishment and bioanalysis of biomarkers in support of drug development. Bioanalysis 4(15), 1883-1894 (2012).

42 Hougton R, Gouty D, Allinson J et al. Recommendations on biomarker bioanalytical method validation by GCC. Bioanalysis 4(20), 2439-2446 (2012).

43 Fraser S, Fleener C, Ogborne K, Soderstrom C. When close is not close enough: a comparison of endogenous and recombinant biomarker stability samples. Bioanalysis $7(11)$, 1355-1360 (2015).

44 Fraser S, Soderstrom C. Due diligence in the characterization of matrix effects in a total IL-13 Singulex ${ }^{\mathrm{TM}}$ method. Bioanalysis 6(8), 1123-1129 (2014).

45 Litwin V, Stewart J, Green C. Receptor occupancy by flow cytometry. Special issue: receptor occupancy by flow cytometry, cytometry part B: clinical cytometry 90B, 108 (2016).

46 Stewart J, Green C, Jones N et al. Overview of flow cytometry receptor occupancy assays and their utility in drug development. Special issue: receptor occupancy by flow cytometry, cytometry part B: clinical cytometry 90B, 110 (2016).

47 Green C, Stewart J, Högerkorp C et al. Recommendations for the development and validation of flow cytometry-based receptor occupancy assays. Special issue: receptor occupancy by flow cytometry, cytometry part B: clinical cytometry 90B, 141 (2016).

48 Sommer U, Morales J, Groenewegen A et al. Implementation of highly sophisticated flow cytometry assays in multi-center clinical studies: considerations and guidance. Bioanalysis 7(10), 1299-1311 (2015).

49 Barnard RM. Flow cytometry: a flexible tool for biomarker research. Bioanalysis 4(20), 2471-2483 (2012).

50 Wu DY, Patti-Diaz L, Hill CG. Development and validation of flow cytometry methods for pharmacodynamic clinical biomarkers. Bioanalysis 2(9), 1617-1626 (2010).

51 O'Hara DM, Xu Y, Liang Z et al. Recommendations for the validation of flow cytometric testing during drug development: II assays. J. Immunol. Methods 363, 120-134 (2011).

52 Green C, Brown L, Stewart J et al. Recommendations for the validation of flow cytometric testing during drug development: I instruments. J. Immunol. Methods 363 , 104-119 (2011).

53 Brown L, Green C, Jones N et al. Recommendations for the evaluation of specimen stability for flow cytometric testing during drug development. J. Immunol. Methods 418, 1 (2015).

54 H52-A2 | Red Blood Cell Diagnostic Testing Using Flow Cytometry (2nd Edition).

55 H42-A2 | Enumeration of Immunologically Defined Cell Populations by Flow Cytometry (2nd Edition).

56 H43-A2 | Clinical Flow Cytometric Analysis of Neoplastic Hematolymphoid Cells (2nd Edition).

57 Wood B, Jevremovic D, Bene MC et al. Group IIW. Validation of cell-based fluorescence assays: practice guidelines from the ICSH and ICCS - part V - assay 
performance criteria. Cytometry B Clin. Cytom. 84, 315-323 (2013).

58 Davis BH, Dasgupta A, Kussick S et al. Group IIW. Validation of cell-based fluorescence assays: practice guidelines from the ICSH and ICCS - part II - preanalytical issues. Cytometry B Clin. Cytom. 84, 286-290 (2013).

59 Davis BH, McLaren CE, Carcio AJ et al. Determination of optimal replicate number for validation of imprecision using fluorescence cell-based assays: proposed practical method. Cytometry B Clin. Cytom. 84, 329-337 (2013).

60 Tanqri S, Vall H, Kaplan D, Hoffman B, Purvis N, Porwit A, Hunsberger B, Shankey TV, Group IIW. Validation of cellbased fluorescence assays: practice guidelines from the ICSH and ICCS - part III - analytical issues. Cytometry B Clin. Cytom. 84, 291-308 (2013).

61 Hill C, Litwin V. Whole-blood lymphocyte immunophenotyping using cell surface markers by flow cytometry. In: Clinical Microbiology Procedures Handbook (4th Edition). Leber AL (Ed.). ASM Press, Washington, DC., p 11.13.1.1-11.13.2.5, doi: 10.1128/9781555818814. ch11.13.

62 Cytobank. www.cytobank.org

63 Jani D, Allison J, Berisha F et al. Recommendations for use and fit-for-purpose validation of biomarker multiplex ligand binding assays in drug development. AAPS J. 18(1), 1-14 (2015).

64 Japanese Ministry of Health, Labour and Welfare. Guideline on Bioanalytical Method (Ligand Binding Assay) Validation in Pharmaceutical Development. Japan (2014).

65 Thway TM, Wang J, Brunner LA et al. Model-based strategy for bioanalytical method comparison: measurement of a soluble ligand as a biomarker. J. Pharm. Biomed. Anal. 58, 65-70 (2012).

66 Yohrling J. Ligand binding assays: risk of using a platform supported by a single vendor. Bioanalysis 1(3), 629-636 (2009).
67 Müller D, Kontermann RE. Bispecific antibodies for cancer immunotherapy. BioDrugs 24(2), 89-98 (2010).

68 Chames P, Batya D. Bispecific antibodies for cancer therapy: the light at the end of the tunnel? mAbs 1(6) 539-547(2009).

69 AlDeghaither D, Smaglo BG, Weiner LM. Beyond peptides and $\mathrm{mAbs}$ - current status and future perspectives for biotherapeutics with novel constructs. J. Clin. Pharm. 55(S3), S4-S20 (2015).

70 Heinrich J, Staack RF, Stubenrauch KG, Papadimitriou A. Proposal for a harmonized descriptive analyte nomenclature for quantitative large-molecule bioanalysis. Bioanalysis 7(24), 3057-3062 (2015)

71 Staack RF, Jordan G, Viert M et al. Quantification of a bifunctional drug in the presence of an immune response: a ligand-binding assay specific for 'active' drug. Bioanalysis 7(24), 3097-3106 (2015).

72 Schick E, Staack RF, Haak M et al. Validation of a ligandbinding assay for active protein drug quantification following the 'free analyte QC concept'. Bioanalysis doi:10.4155/bio2016-0172 (2016) (Epub ahead of print).

73 DeSilva B, Smith W, Weiner R et al. Recommendations for the bioanalytical method validation of ligand-binding assays to support pharmacokinetic assessments of macromolecules. Pharm. Res. 20 (11), 1885-1900 (2003).

74 Viswanathan CT, Bansal S, Booth B et al. Quantitative bioanalytical methods validation and implementation: best practices for chromatographic and ligand binding assays. Workshop/Conference Report. AAPS J. 9(1), e30-e42 (2007)

75 Steve Lowes, Richard LeLacheur, Ronald Shoup et al. Recommendations on incurred sample stability (ISS) by GCC. Bioanalysis 6(18), 2385-2390 (2014). 